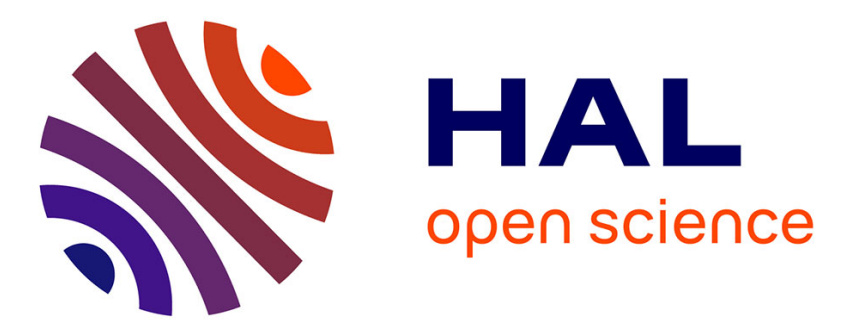

\title{
Internal waves generated by the wake of Gaussian hills
} Pascal Dupont, Yassine Kadri, Jean-Marc Chomaz

\section{To cite this version:}

Pascal Dupont, Yassine Kadri, Jean-Marc Chomaz. Internal waves generated by the wake of Gaussian hills. Physics of Fluids, 2001, 13 (11), pp.3223-3233. 10.1063/1.1406936 . hal-01025348

\section{HAL Id: hal-01025348 \\ https://hal-polytechnique.archives-ouvertes.fr/hal-01025348}

Submitted on 11 Sep 2014

HAL is a multi-disciplinary open access archive for the deposit and dissemination of scientific research documents, whether they are published or not. The documents may come from teaching and research institutions in France or abroad, or from public or private research centers.
L'archive ouverte pluridisciplinaire HAL, est destinée au dépôt et à la diffusion de documents scientifiques de niveau recherche, publiés ou non, émanant des établissements d'enseignement et de recherche français ou étrangers, des laboratoires publics ou privés. 


\section{AIP | Physics of Fluids}

\section{Internal waves generated by the wake of Gaussian hills}

Pascal Dupont, Yassine Kadri, and Jean-Marc Chomaz

Citation: Physics of Fluids (1994-present) 13, 3223 (2001); doi: 10.1063/1.1406936

View online: http://dx.doi.org/10.1063/1.1406936

View Table of Contents: http://scitation.aip.org/content/aip/journal/pof2/13/11 ?ver=pdfcov

Published by the AIP Publishing

\section{Articles you may be interested in}

An Experimental Comparison of Internal Waves Generated by a Moving Hemispheroid or Spheroid Model Respectively in a Stratified Fluid Tank

AIP Conf. Proc. 1376, 291 (2011); 10.1063/1.3651900

Spatial characterization of vortical structures and internal waves in a stratified turbulent wake using proper orthogonal decomposition

Phys. Fluids 22, 086601 (2010); 10.1063/1.3478837

Elliptical-inertial instability of rotating Karman vortex streets

Phys. Fluids 17, 066602 (2005); 10.1063/1.1937348

The generation of internal waves by a towed sphere and its wake in a thermocline

Phys. Fluids 9, 3353 (1997); 10.1063/1.869448

A model of wave dynamics in the far wake of a cylinder

Phys. Fluids 9, 1969 (1997); 10.1063/1.869316

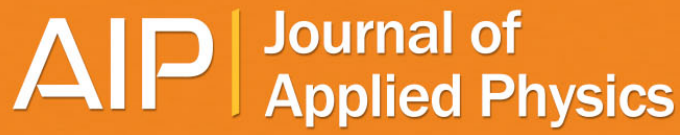

Journal of Applied Physics is pleased to announce André Anders as its new Editor-in-Chief 


\title{
Internal waves generated by the wake of Gaussian hills
}

\author{
Pascal Dupont \\ LTI, CNRS-EPUN, BP 90604, 44306 Nantes Cedex 3, France \\ and LEGI, CNRS-INPG-UJF, BP53, 38041 Grenoble Cedex 9, France \\ Yassine Kadri \\ SPEA/GMEI/CNRM, Météo-France, 42 av. G. Coriolis, 31057 Toulouse Cedex, France \\ Jean-Marc Chomaz \\ LADHYX, CNRS Ecole Polytechnique, 91128 Palaiseau-Cedex, France
}

(Received 27 February 2001; accepted 28 June 2001)

\begin{abstract}
Depending on their structure and dynamics, the wakes of various obstacles can generate different kinds of internal waves in stratified fluids. Experiments on waves emitted by three-dimensional Gaussian models towed uniformly in a linearly stratified fluid were carried out. Beyond a critical value $\mathrm{Fr}_{c}$ of the Froude number Fr, the developed wake was observed to radiate an internal wave field shorter than the lee waves of the hill. The emergence of such waves is correlated with the periodic shedding of three dimensional vortical structures at $\mathrm{Fr}>\mathrm{Fr}_{c}$. Measurements show that the wavelengths of these short waves are constant in space and time, and proportional to Fr. Their phase and group velocities are proportional to the coherent structure velocity which is estimated from velocity measurements inside the wake. All those spatio-temporal characteristics prove that these short waves are generated by the displacement of the coherent structure inside the wake. (C) 2001 American Institute of Physics. [DOI: 10.1063/1.1406936]
\end{abstract}

\section{INTRODUCTION}

Stratified flows over a three-dimensional obstacle have been extensively studied for geophysical and industrial applications using the equivalent laboratory motion of a moving body in a stratified medium. Although the lee waves generated by the body itself are well understood, the internal waves generated by the turbulent wake of a body are poorly described and even now cannot be fully predicted. Lin and $\mathrm{Pao}^{1}$ have demonstrated that internal waves generated by wakes depend crucially on the nature of the moving body which creates different kinds of wakes: A towed grid occupying the full towing tank section produces a stratified turbulence which collapses with an abrupt restriction of the vertical turbulent motion (decrease of the Thorpe length scale); in the case of a grid with a finite area, the turbulent wake surrounded by the stratified medium collapses vertically and spreads horizontally (see Merrit ${ }^{2}$ ) emitting a complex wave field; at small Froude number, a towed two-dimensional object produces nonlinear internal waves and an upstream wake due to the blocked fluid; a self-propelled axisymmetric body produces a wake very similar to the small grid one (see Schooley and Hughes ${ }^{3}$ ) with similar internal waves; and a towed axisymmetric object produces a wake dominated by coherent structures whose upward and downward moving parts collapse individually (see Bonneton et al..$^{4}$ ) emitting impulsive wave fields. In the relevant case of an axisymmetric body, Gilreath and Brandt ${ }^{5}$ have analyzed the wake in order to point out the various internal wave sources associated with the moving body and its wake. In the case of a self-propelled body, they have distinguished the body source, the propeller source, the mean wake collapse source and the turbulence. The first three sources are fixed with the moving body and will be called stationary sources. Gilreath and Brandt ${ }^{5}$ and Bonneton et al. ${ }^{4}$ have clearly shown that the last source is very important at large Froude number and the resulting internal wave field cannot be fully calculated because of the random behavior of the source. In the case of a towed sphere, Bonneton et al. ${ }^{4}$ have explained the wave emission mechanism by turbulence: Each large-scale structure of the wake induces upward (and downward) boundary convolutions which collapse individually and radiate an impulsive wave field. When these intrusions, i.e., coherent structures, are fairly periodic, they generate an asymptotic wave field which has been described by both Gilreath and Brandt ${ }^{5}$ and Voisin. ${ }^{6}$ Furthermore, Lin et al. ${ }^{7}$ and Sysoeva and Chashechkin ${ }^{8}$ have concluded from measurements that the coherent structures of the wake represent the basic source of internal waves in the wake without explaining clearly the wave generation mechanism.

In this paper, we demonstrate from experimental evidence a mechanism of internal wave generation by wakes, more specifically, that the waves are generated by the selfadvection of each coherent structure viewed as a solid moving body. This conclusion is based on experiments of stratified flows over a Gaussian hill at Froude number Fr $=U /(N h) \in[1.5,10]$ and Reynolds number $\operatorname{Re}=2 L U / v$ $\in[3300,19400]$ with $U$ the hill velocity, $h$ and $L$ its height and length, $N=\left(-g / \rho \partial_{z} \rho\right)^{1 / 2}$ the Brunt-Väisälä frequency and $v$ the kinematic viscosity of the fluid. Our experiments were focused on the internal waves radiated by the wake of Gaussian forms of aspect ratios $h / L=0.28$ and 0.8. In Sec. II, we present the experimental apparatus, the wake structure and the observed wave field. The main result is the visualization of surfaces of constant phase and their interpretation using linear theory with the different hypotheses about wave 


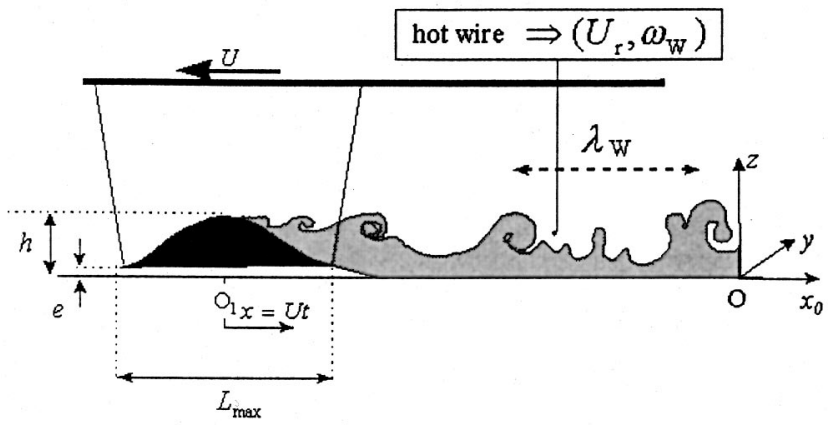

FIG. 1. Experimental apparatus similar to the one used by Kadri et al. (Ref. 11).

emission. Section III discusses the various characteristics of the wave sources in the wake of a three-dimensional object. They are classified in four groups according to different wave emission mechanisms producing waves with similar characteristics. The stationary sources generate waves called Body Lee Waves (BLW) which are similar to the well-known lee waves. They include the stationary flow around the body and possibly the propeller perturbation and the mean collapse of the mixed part of the wake which is particularly important when a propeller mixes the fluid behind the object. Oscillating sources generate waves called Oscillating Waves (OW) which include all periodic phenomena in the wake and in particular the oscillation of the recirculating zone and the collective effect of the periodic collapse of the upward and downward moving parts of the large coherent structures in the wake. The collapse of all turbulent structures emits socalled Random Waves (RW), which are a superposition of impulsive wave fields. The fourth gravity wave source that we have clearly identified is associated with the coherent structures' self-advection. The radiated waves are called Coherent Structure Lee Waves (CSLW) because of their resemblance to the lee waves generated by a rigid body moving at the self-induced velocity of the coherent structure. In Sec. IV, the characteristics of the four wave fields are compared to the experimental wave field with respect to the shape of surfaces of constant phase, the wavelength, the wave frequency (related to the phase velocity), and the vertical group velocity.

\section{EXPERIMENTS}

\section{A. Apparatus}

The experiments were performed in a water towing tank of Météo-France in Toulouse $\left(0.5 \times 0.5 \times 4 \mathrm{~m}^{3}\right)$, made entirely of glass for visualization purposes. The tank was filled with a linearly stratified fluid obtained by the Oster ${ }^{9}$ two-tank method. In the actual experiments, the Brunt-Väisälä frequency was $N=1.5 \mathrm{rad} / \mathrm{s}$. The Gaussian model is supported by five $0.1 \mathrm{~mm}$ diam wires at a distance, $e$, from the bottom which varied from 2 to $3 \mathrm{~mm}$ (see Fig. 1). Results obtained with two Gaussian models are reported here. Their shape is given by

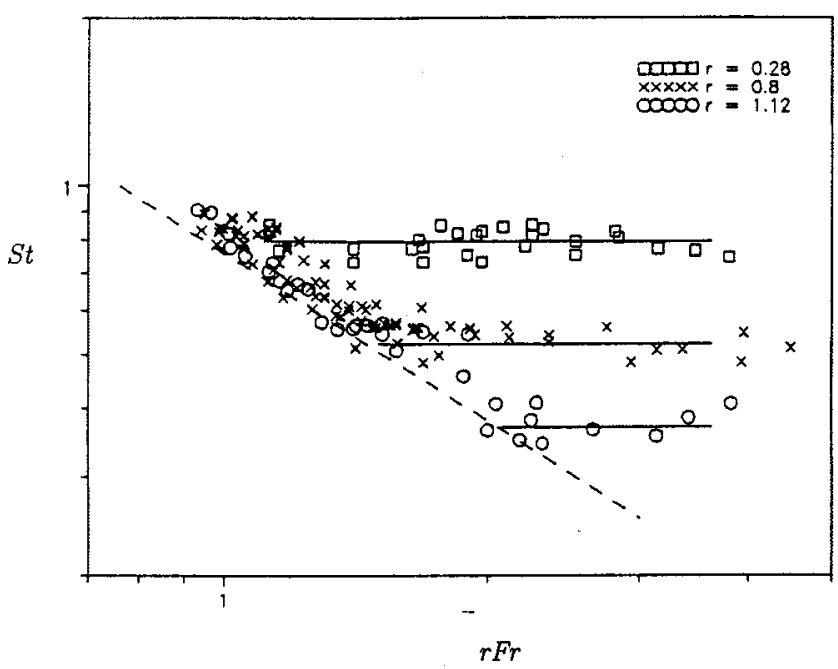

FIG. 2. Experimental Strouhal number St of the sinuous instability of the wake vs Froude number Fr. Measurements were made with a conductivity probe placed in the intermittent zone of the near wake for two aspect ratios $h / L=0.28$ (triangle) and $h / L=0.8$ (square) (from Kadri et al. (Ref. 11)).

$$
z=\left\{\begin{array}{l}
h \exp \left(-\frac{x^{2}+y^{2}}{L^{2}}\right) \quad \text { for }\left(x^{2}+y^{2}\right)^{1 / 2}<L_{\max }, \\
0 \quad \text { otherwise }
\end{array}\right.
$$

the characteristic lengths are $L=4.8 \mathrm{~cm}, L_{\max }=7.5 \mathrm{~cm}$ and $h / L=0.28$ for the first model; and $L=3.4 \mathrm{~cm}, L_{\max }$ $=6.07 \mathrm{~cm}$ and $h / L=0.8$ for the second one. In order to visualize the internal wave field in horizontal planes, we use the method described in Chomaz et al. ${ }^{10}$ which consists of using a horizontal laser sheet to illuminate a horizontal fluorescent dyed layer. The camera placed below the tank records the time evolution of the fluorescent light as internal waves displace the dyed layer with respect to the laser sheet. In all images shown, $N t=0$ refers to the time when the model center disappears from the visualization domain and distances are expressed in the frame moving with the body $x$ $=U t$. This wave visualization has been supplemented by conductivity probe measurements, with probes moving with the obstacle away from the wake, which detect only the unsteady internal waves and not the body lee waves (BLW). Additional velocity measurements are performed with moving hot wires inside the wake in order to determine the relative velocity, $U_{r}$, and thus a characteristic velocity $U_{\mathrm{CS}}$ of the visualized coherent structures (CS) and also to measure the vortex shedding frequency $\omega_{W}$ in the wake.

\section{B. The wake study}

Different regimes of the stratified flow past Gaussian models was studied experimentally by Kadri et al. ${ }^{11}$ Threedimensional transitions were shown to depend mainly on the Froude number $\mathrm{Fr}=U /(N h)$ and the aspect ratio $h / L$, but, to a lesser extent, on the Reynolds number $\operatorname{Re}=2 L U / v$ if $\operatorname{Re}$ is large enough $\left(\operatorname{Re}>10^{3}\right)$. Beyond the critical value of the Froude number $\mathrm{Fr}_{c}=L / h$, a global wake oscillation takes place and causes periodic shedding of a large hairpin vortices at the frequency $\omega_{W}(\mathrm{in} \mathrm{rad} / \mathrm{s})$. Measured Strouhal number $\mathrm{St}=\omega_{W} L / \pi U$ versus Fr are presented on Fig. 2 where $\omega_{W}$ is 


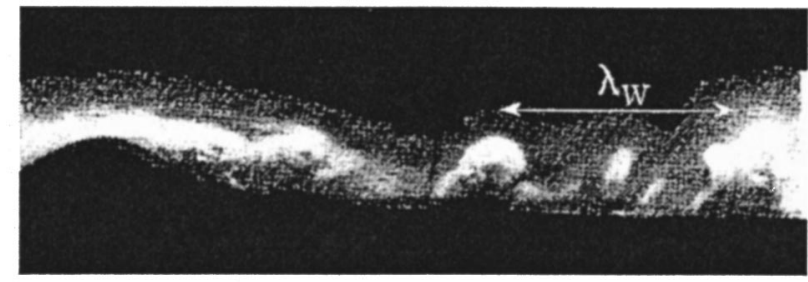

(a)

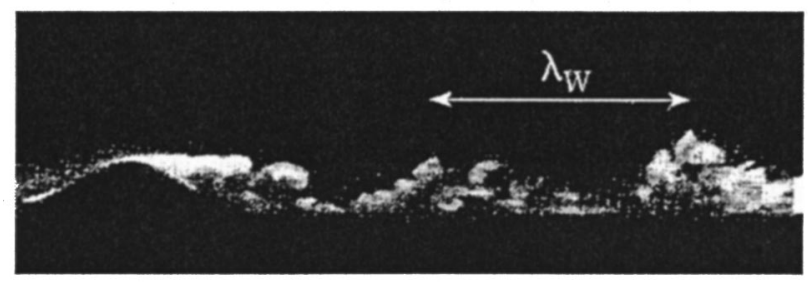

(b)

FIG. 3. Laser induced visualization in the vertical median plan of the wake for the hill with an aspect ratio $h / L=0.8$ and at Froude number $F r=1.5$ (a) and $\mathrm{Fr}=3$ (b). The length of images correspond to $N t=N x / U=7.7$ for (a) and $N t=4.2$ for (b) and the Reynolds number is related to the Froude number: $\mathrm{Re}=2200 \mathrm{Fr}$.

obtained by moving hot wires measurements (see Fig. 1). We observe that St rapidly reaches its neutral value which is close to 0.88 for $h / L=0.28$ and to 0.55 for $h / L=0.8$. For $h / L=0.8$ and $\mathrm{Fr}=1.5$ and 3 , the side views (Fig. 3 ) of the flows exhibit a fairly regular large-scale vortices with a spatial periodicity $\lambda_{W}$. The maximum vertical extension of these coherent structures is larger than $h$. For both Gaussian hills used, we carried out measurements of axial velocity $U_{r}$ with the same hot wires moving with the obstacle centered in the lee at streamwise location $x=13 h$ and elevation $z=h / 2$ where coherent structures are always present, fully developed and not yet collapsed. $U_{r}$ being a relative velocity, the mean coherent structure velocity in the laboratory frame $U_{\mathrm{CS}}$ is defined by $U_{\mathrm{CS}}=U-\overline{U_{r}}$ with $\overline{U_{r}}$ the time average of $U_{r}$ over the available signal (Fig. 4). Considering $U_{\mathrm{CS}}$ as the coherent structure advection velocity is not obvious, we call it so because this mean velocity objectively characterizes the

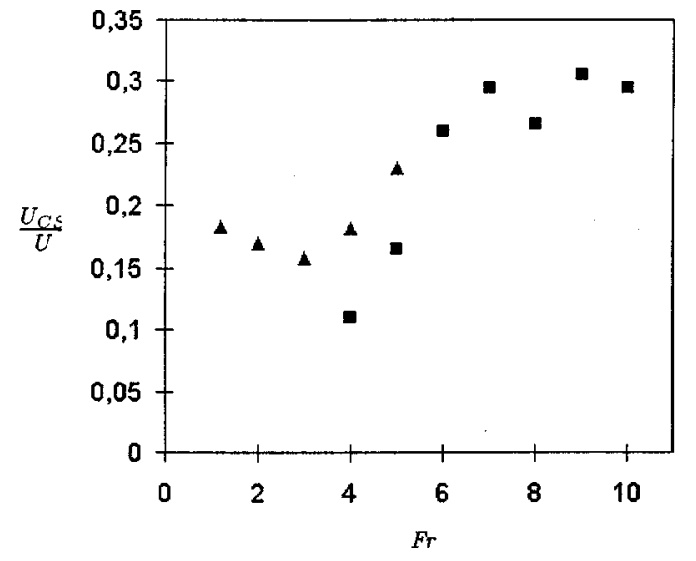

FIG. 4. Experimental mean velocity inside the wake $U_{\mathrm{CS}} / U$ vs Fr for two aspect ratios $h / L=0.28$ (square) and $h / L=0.8$ (triangle). $U_{\mathrm{CS}}$ is obtained from probe moving with the obstacle and located at $x / h=13$ and $z=h / 2$. wake activity. Furthermore in all the experiments performed we found $U_{\mathrm{CS}} \approx 0.2 U$ which is in good agreement with value of $U_{\mathrm{CS}}$ deduced from measurements of the wake frequency and of the axial periodicity (from visualization) through $U$ $-U_{\mathrm{CS}} \approx \lambda_{W} \omega_{W} / 2 \pi$ (data not reported here). This relation for $U_{\mathrm{CS}}$ is corroborated by directly estimating the axial motion of coherent structures from visualization. The lifetime of coherent structures is estimated from images similar to Fig. 3, but taken further downstream. On Fig. 3 the collapse of the coherent structures has not yet occurred. The observed collapse time $t_{c}\left(N t_{c}=N x_{c} / U \geqslant 10\right)$ is larger than in the case of the turbulente wake of a sphere $\left(N t_{c} \sim 2.5\right)$. Actually, the published values of $N t_{c}$ seem to vary with the mechanism of the vortical structures generation. For an oscillating sphere Lin et al. ${ }^{12}$ found $N t_{c} \approx 4-7$, and for a single vertical vortex rings Van Atta and Hopfinger ${ }^{13}$ found $N t_{c} \approx 5-11$.

\section{Gravity wave field}

Figures 5 and 6 present a series of snapshots of the wave field in a horizontal plane, taken at a fixed location in the laboratory frame and at different times $t$ after the passage of the hill. On each picture $x$ is expressed in the frame moving with the hill using the Taylor hypothesis $x=U t$. Figures 5 and 6 correspond to the same model $(h / L=0.8)$ and the same vertical distance of the visualization plane from the ground $(z / h=5)$ but the Froude numbers are $\mathrm{Fr}=1.5$ for Fig. 5 and $\mathrm{Fr}=3$ for Fig. 6. Surprisingly the wave fields for this different Fr are very similar (Figs. 5 and 6) whereas if only lee waves were considered their should not. As we will demonstrate the results illustrate the transition between a wave field dominated by waves generated by the obstacle noted BLW for Body Lee Wave at $\mathrm{Fr}=1.5$, to a wave field composed almost exclusively of waves emitted by the displacement of coherent structure noted CSLW for Coherent Structure Lee Waves (CSLW) at Fr $=3$.

For $\mathrm{Fr}=1.5$, the waves immediately downstream and above the obstacle are the well-known body lee waves (BLW) whose characteristics are recalled in the next section. In the last image and in the central zone, we can nearly distinguish short waves which are very weak at $\mathrm{Fr}=1.5$. These waves have a wavelength $\lambda_{x}$ and a phase velocity $80 \%$ smaller than that of the lee waves. The emergence of this second wave field is correlated to the emergence of the organized three-dimensional structures in the wake that indeed move at $20 \%$ of the hill velocity. For Fr $\geqslant 3$, this second type of wave is dominante. They still possess a wavelength and a phase velocity $80 \%$ smaller than that of the lee wave and we will call them short waves in comparison to the lee waves even though their wavelength may be large compared to $L$ when $U$ is large. Their lines of constant phase are drawn on the right cartoons of Fig. 6. For this relatively large Froude number, the BLW have a wavelength about the image size. They are not associated with franges on a single image but they induce periodic change in the mean brightness between the left and right side of the images (Fig. 6) that modulates the short wave pattern. In this figure constant phase lines of short waves present a $\mathrm{V}$-shape which closes with time as do lee waves (Fig. 5). Therefore, it is tempting to associate these 

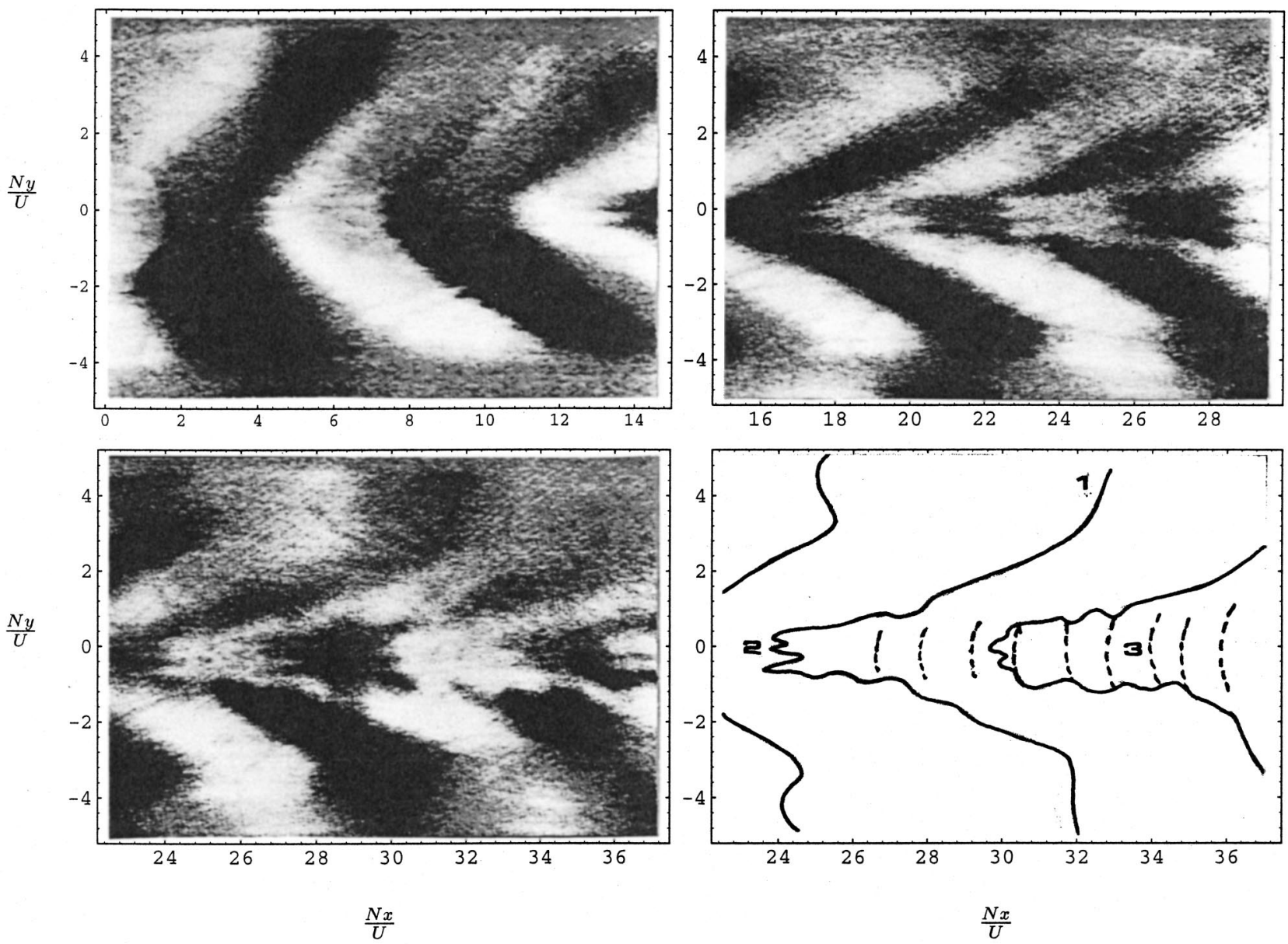

FIG. 5. Internal waves generated by the motion of the model $h / L=0.8$ at $z=5 h$ and for $\operatorname{Fr}=1.5$. The value $N x / U$ gives the time elapsed since the hill has passed. The last image shows lines of constant phase corresponding to the Body Lee Waves (1), the perturbation due to the sustaining wires (2) and the short internal waves (3) generated by the wake which are more clearly on a movie. Dashed lines indicate the weakness of the observed lines of constant phase.

waves to the coherent structure that would act as a body moving $80 \%$ smaller than the hill.

\section{INTERNAL WAVE SOURCES IN WAKES}

In order to conforte this idea of wave emitted by coherent structure as if they were solid body, we try in the present section to classified and describe the wave field associated with different source active in the wake of a hill. Each emission mechanism is illustrated in Fig. 7 by the wave pattern in the central plane. We describe first the waves generated by stationary sources fixed in the moving frame like the hill but also like the mean wake collapse. These are called Body Lee waves (BLW). Secondly, we summarize the characteristics of the waves emitted by oscillating source (noted OW for Oscillating Waves) such as the oscillation of the recirculating zone behind the body. Then we draw the wave fields generated by a single organized structure: its mean advection induces Coherent Structure Lee Waves (CSLW) and its collapse generates Random Waves (RW). Finally we present the result of the superposition of wave fields due to several synchronized individual sources. These collective effects induce particular wave fields that we name Resonant CSLW (RCSLW) for the collective advection of coherent structures and OW for their periodical collapse. In each case the phase function is called and is used to obtain the phase velocity $\mathbf{c}_{\phi}$, the relative frequency $\omega_{r}$ (in the moving frame), the absolute frequency $\omega_{a}$ (in the laboratory frame) and the group velocity $\mathbf{c}_{g}$ using the classical relations $\omega_{a}=\partial_{t} \Phi, \mathbf{k}=\nabla_{\mathbf{r}} \Phi, \omega_{r}$ $=\omega_{a}-\mathbf{k} \cdot \mathbf{U}, \mathbf{c}_{\phi}=\omega \mathbf{k} / k^{2}, \mathbf{c}_{g}=\nabla_{\mathbf{k}} \omega_{a}$.

\section{A. Stationary sources (BLW)}

Gilreath and Brandt ${ }^{5}$ have split the perturbations induced by a self-propelled body into three wave sources: The body itself, the mean wake collapse and the propeller. They emit wave similar to lee waves (BLW), the characteristics of which are well predicted by the linear theory of a moving point source developed by Lighthill ${ }^{14}$ and Miles ${ }^{15}$ among others. In the moving coordinate system $(x, y, z)$ of Fig. 7(a) with the point source at the origin, the phase function is stationary

$$
\Phi_{\mathrm{BLW}}(x, y, z)=\frac{N}{U}|z|\left(\frac{x^{2}+y^{2}+z^{2}}{y^{2}+z^{2}}\right)^{1 / 2},
$$

the wave amplitude being related to the spectrum of each source (see Miles ${ }^{15}$ ). Actually the difficulty is to construct the spectrum of each equivalent source of waves. In each 

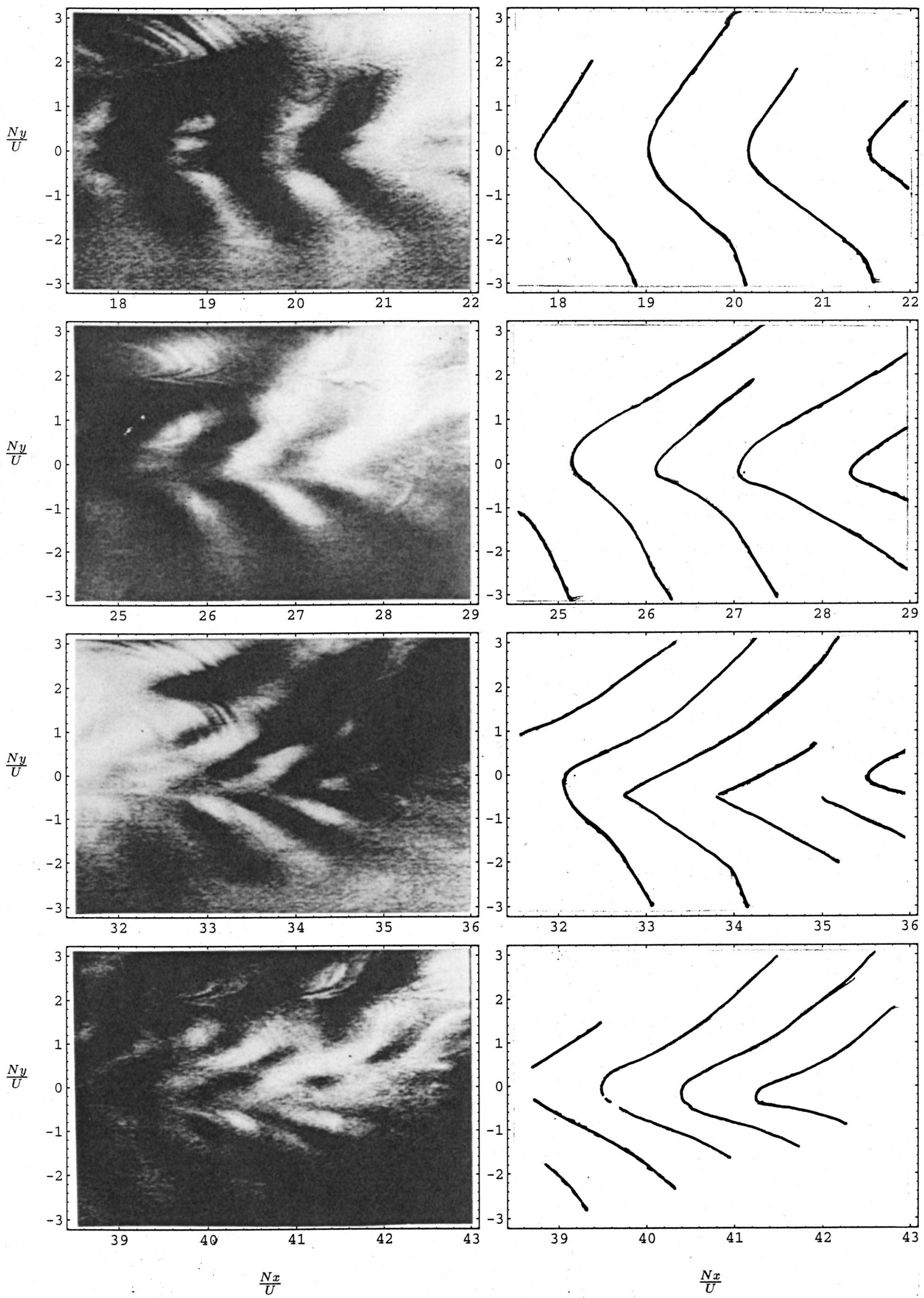

FIG. 6. Internal wave field visualized for $h / L=0.8$ and $\mathrm{Fr}=3$ at $z=5 h$ and different time with the lines of constant phase on the right. 


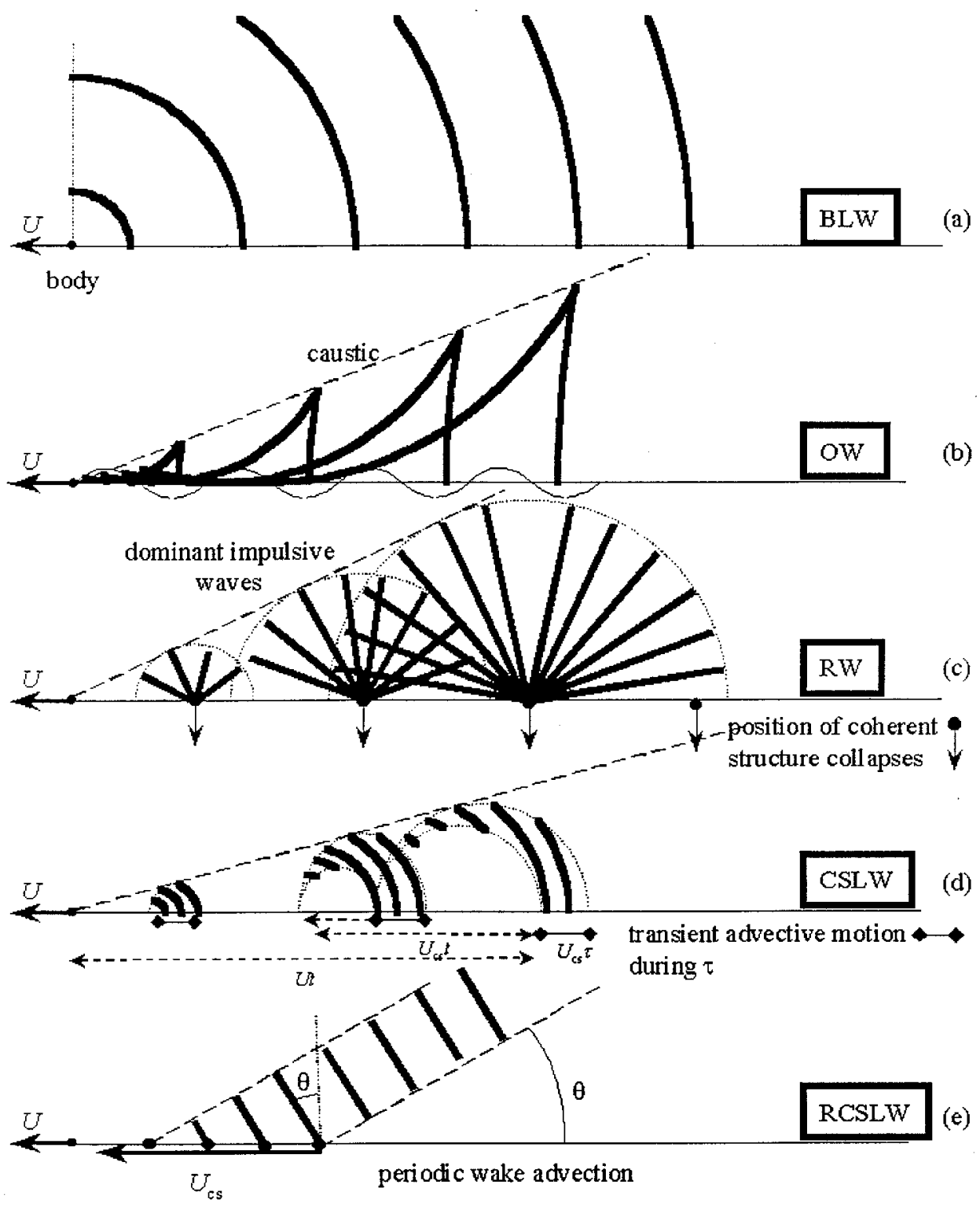

FIG. 7. Comparison between the lines of constant phase of the various internal wave components in the center plane. The dashed lines represent the wave front of the dominent waves or the caustic of the wave field. In (c), the arrows indicate the position of the coherent structure collapses; in (d) the transient advective motion of each coherent structure is represented as continuous line for the active emission period and as dotted line for their virtual motion after their collapse; in (e) only dominant internal waves are represented.

(d) (e)

horizontal plane $z=$ const, and far downstream (i.e., at large $x$ ) the $x$-wavelength tends rapidly to $2 \pi \mathrm{Fr} h$, and the hyperbola are closing up (see Hopfinger et al. ${ }^{16}$ ).

\section{B. Oscillating and translating source (OW)}

Dupont and Voisin ${ }^{17}$ studied the Oscillating Waves (OW) generated by a sphere translating horizontally at the velocity $U$ and oscillating vertically at the frequency $\omega_{W}$ for the oscillating sources of the wake [Fig. 7(b)]. They described several components connected to distinct values of their relative frequency $\xi_{i}=\omega_{i} / N$ in the moving frame of reference. Here again the phase variation of these waves can be calculated

$\Phi_{\mathrm{OW}}=\omega_{W} t+\frac{N}{U} \frac{|z \sin \phi|}{\xi_{i}^{2}} \frac{\left(\xi_{i}-\omega_{W} / N\right)^{2}}{\left(\sin ^{2} \phi-\xi_{ \pm}^{2}\right)} \operatorname{sign}\left[\xi_{i}-\omega_{W} / N\right]$, with $\xi_{i}$ the roots of the polynomial equation obeying a radiation condition (see Lighthill ${ }^{14}$ )

$$
\begin{aligned}
& \xi^{6}-\xi^{4} \cos ^{2} \theta \sin ^{2} \phi-2 \frac{\omega_{W}}{N} \xi^{3} \sin \theta \sin ^{2} \phi+\frac{\omega^{2}}{N^{2}} \sin ^{2} \theta \sin ^{4} \phi \\
& \quad=0,
\end{aligned}
$$

where $(\rho, \theta, \phi)$ are the spherical coordinates $(x=\rho \cos \theta, y$ $=\rho \sin \theta \cos \phi, z=\rho \sin \theta \sin \theta)$.

\section{Individual collapse of the coherent structures (RW)}

Bonneton et al. ${ }^{4}$ demonstrated that the individual vertical collapse of each turbulent burst generates an impulsive wave field [Fig. 7(c)] which supersedes lee waves for Fr $>4$ (and large Re). Actually they observed a superposition of multiple impulsive wave fields which they called Random 


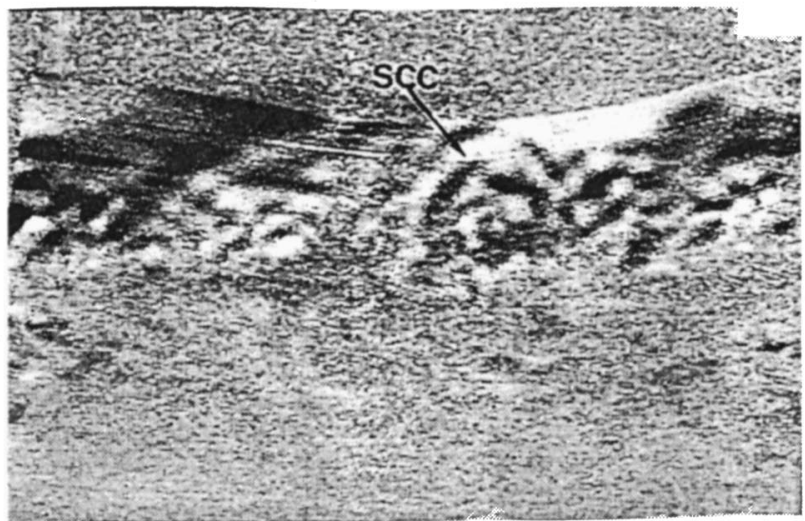

(a)

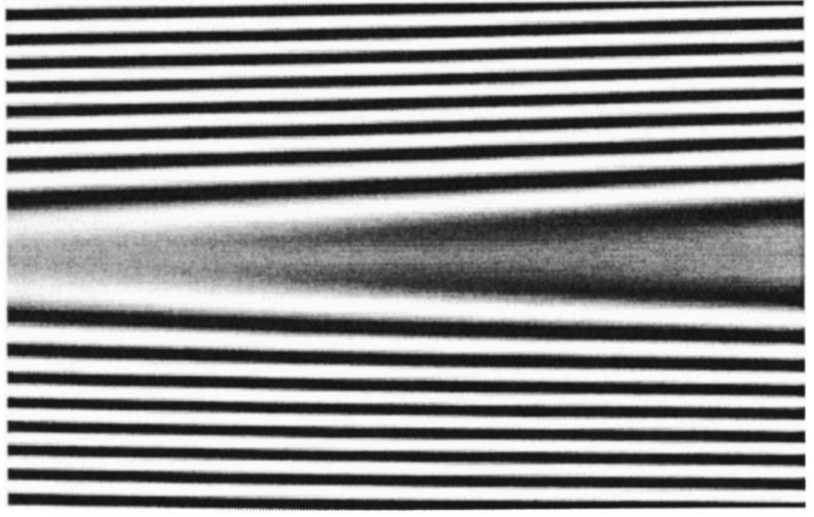

(c)

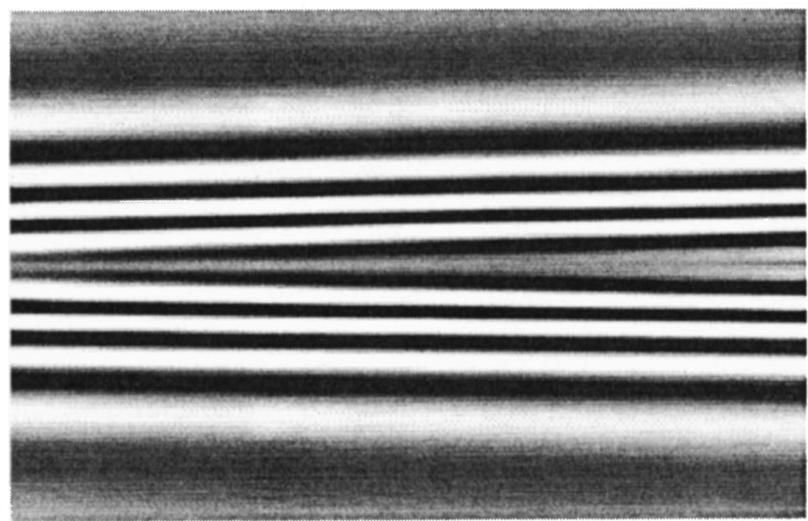

(b)

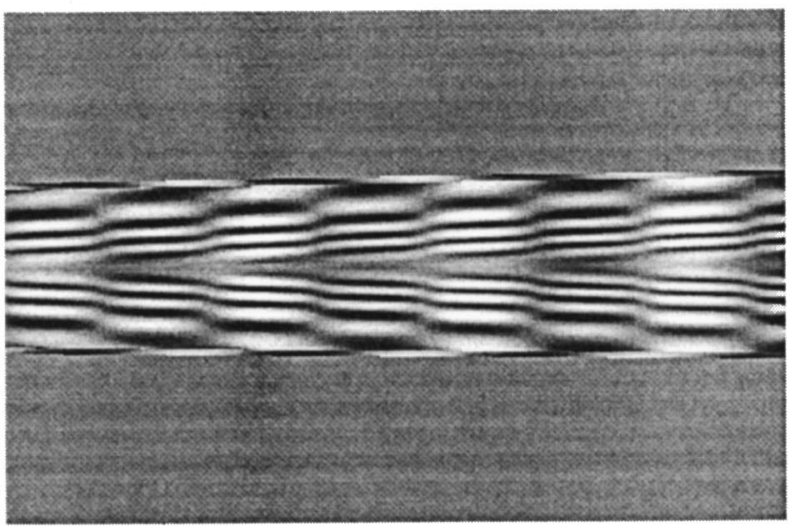

(d)

FIG. 8. Comparison between the theoretical wave fields and the random wave field (a) observed by Bonneton $e t$ al. (Ref. 4) for $\mathrm{Fr}=5$ and $z / h=3$ : (b) the Body Lee Waves (BLW), (c) the Coherent Structure Lee Waves (CSLW), and (d) the Oscillating Waves.

Waves (RW) according to the terminology of Gilreath and Brandt. ${ }^{5}$ The phase variation of the resulting waves is then $\Phi_{\mathrm{RW}}$ described by Lighthill $^{14}$

$$
\Phi_{\mathrm{RW}}=N t \frac{z^{\prime}}{\sqrt{x^{\prime 2}+y^{\prime 2}+z^{\prime 2}}},
$$

with $\left(x^{\prime}, y^{\prime}, z^{\prime}\right)$ the frame fixed with the fluid and $t$ the elapsed time since the single impulse at $t=0$ and at $x^{\prime}=y^{\prime}$ $=z^{\prime}=0$. The wave field experimentally observed corresponds then to the superposition of many impulsive wave fields generated at different locations and different times. In horizontal plane the lines of constant phase is organized in superposed concentric circles [see Fig. 8(a)].

\section{Individual advection of the coherent structures (CSLW)}

Sysoeva and Chashechkin ${ }^{8,18}$ described very briefly a coherent internal wave field generated by the wake's vortical structures for $\mathrm{Fr}>4$, but $\mathrm{Re} \leqslant 2000$. In this range of parameters, it seems that the coherent structures move horizontally at a velocity $U_{\mathrm{CS}}$ and generate their own "attached internal waves" as does a solid body [Fig. 7(d)]. Each coherent structure generates attached internal waves here called CSLW field with a phase variation $\Phi_{\mathrm{CSLW}}$ given by

$$
\Phi_{\mathrm{CSLW}}\left(x^{\prime \prime}, y^{\prime \prime}, z^{\prime \prime}\right)=\frac{N}{U_{\mathrm{CS}}}\left|z^{\prime \prime}\right|\left(\frac{x^{\prime \prime 2}+y^{\prime \prime 2}+z^{\prime \prime 2}}{y^{\prime \prime 2}+z^{\prime \prime 2}}\right)^{1 / 2},
$$

with $\left(x^{\prime \prime}, y^{\prime \prime}, z^{\prime \prime}\right)$ in the frame moving with the coherent structure. Far downstream the $x$-wavelength tends to $2 \pi \operatorname{Frh} U_{\mathrm{CS}} / U$ and the phase line in horizontal plane are hyperbola that are closing when $x^{\prime \prime}$ and also $x$ increases.

\section{E. Collective effects of coherent structures collapses (OW)}

In order to understand the random internal waves observed by Bonneton et al., ${ }^{4}$ Voisin ${ }^{6}$ proposed a mathematical model of the collective effect of the quasi-periodic collapses of the upward and downward moving parts of the coherent structures. He constructed the coherent part of the superposition of a large number of periodic impulsive wave fields periodically applied in time and space. The equivalent source is then an oscillating (at the frequency $\omega=\omega_{W}$ ) and translating (at the velocity $U$ of the body) source generating a wave field which follows the phase variation of Eq. (3). The cru- 
TABLE I. Axial wavelength of each possible wave component emitted by wake calculated in the center plane $y=0$.

\begin{tabular}{lll}
\hline \hline Source type & Individual effect & Collective effect \\
\hline Stationary sources & $\begin{array}{l}\lambda_{B L W}^{x}=2 \pi U N^{-1}\left(1+z^{2} / x^{2}\right)^{1 / 2} \\
\\
\lambda_{B L W}^{x} \sim 2 \pi U N^{-1} \text { for } x / z \gg 1\end{array}$ \\
Coherent structure & $\lambda_{R W}^{x}=2 \pi(U / N)(h / L)^{3} /\left(2 z^{\prime} x^{\prime 2}\right)$ & \\
Collapse & & $\begin{array}{l}\lambda_{O W}^{x}=2 \pi U N^{-1}\left|\frac{\omega_{W}}{N}-\xi_{ \pm}\right|^{-1} \\
\lambda_{O W}^{w} \sim \lambda w \text { for } \omega_{w} / N \gg 1\end{array}$ \\
Coherent structure & $\lambda_{C S L W}^{x}=2 \pi U_{C S} N^{-1}\left(1+z^{\prime \prime 2} / x^{\prime \prime 2}\right)^{1 / 2}$ & $\lambda_{R C S L W}^{x}=\lambda_{W}$ \\
Advection & $\lambda_{C S L W}^{x} \sim 2 \pi U_{C S} N^{-1}$ for $x^{\prime \prime} / z^{\prime \prime} \gg 1$ & \\
\hline \hline
\end{tabular}

cial parameter in this calculation is the forcing frequency $\omega_{W}$ of the coherent structures empirically related to the flow parameter: $\omega_{W} / N=\pi r \mathrm{St}$ Fr, St being the Strouhal number of the sinuous instability [which is constant for a given geometry and Fr not too small (see Fig. 2)]. Dupont ${ }^{19}$ has demonstrated that the wave front, the wavelength and the wave amplitude predicted by this model are in good agreement with the experiments of Hopfinger et al. ${ }^{16}$ and Bonneton et $a l^{4}$ The $x$-wavelengths are then closed to $\lambda_{W}$ for large $\omega_{W} / N$.

\section{F. Collective effects of coherent structures advection (RCSLW)}

In the model of coherent structures advection, a second collective effect is also possible. Actually periodic coherent structures are advected at nearly the same mean velocity and induces a wave field similar to the moving corrugated wall case [see Fig. 7(e)]. This moving source is an extended one with a streamwise periodicity $\lambda_{W}$ and the resulting wave field could be quite different in amplitude and phase from lee waves generated by a compact moving source. In particular, waves having an axial wavenumber equal to $2 \pi / \lambda_{W}$ are resonant with the source and, if excited, would exhibit a large amplitude. This is the case when $2 \pi U_{\mathrm{CS}} /(N)<\lambda_{W}$, the first term being the shortest $x$-wavelength emitted by a single coherent structure. Then for sufficient $U_{\mathrm{CS}}$ (i.e., sufficient $U$ ), the resulting wave field is dominated by the Resonant Coherent Structure Lee Waves (RCSLW) with the following wave vector (see Dupont ${ }^{19}$ )

$$
\begin{aligned}
& k_{x}=2 \pi / \lambda_{W}, \\
& k_{z}^{2}=\left(k_{x}^{2}+k_{y}^{2}\right) \frac{1-\left(N k_{x} / U_{\mathrm{CS}}\right)^{2}}{k_{x}^{2}} .
\end{aligned}
$$

\section{G. Theoretical summary}

To compare with experiments, we deduce for each wave source the streamwise wavelength which is related to the phase function through $\lambda_{x}=2 \pi\left|\partial_{x} \Phi\right|^{-1}$. In order to interpret the waves generated by the wake, we summarize for each source the associated streamwise wavelength in the center plane $(y=0)$ in Table I. In order to distinguish between the different wave fields and to understand the different mechanisms of emission, we construct in Fig. 7 a comparison between the characteristic lines of constant phase in the center plane $(y=0)$ for each wave component. We emphasize that the origins of the sources in the wake are taken at the body center and this rough approximation changes the real origines of the $x$ and $z$ axes. The four internal waves are quite different even far downstream. In a real experiment, all four components are present and their relative amplitudes depend on the strength of each source.

\section{INTERPRETATION OF THE INTERNAL WAVE VISUALIZATIONS}

\section{A. The shape of surfaces of constant phase}

The BLW of Fig. 5 and the short internal waves of Fig. 6 present typical lee wave parabolic structures. The short waves differ radically from the impulsive wave field, with conical constant phase [see Fig. 7(c)] observed by Bonneton et $a l^{4}$ and drawn in Fig. 8(a). Thus the mechanism of short wave generation is quite different from the impulsive collapse and is observed not far enough from the hill for the collective effect of periodic collapses to be invoked (cf. Dupont ${ }^{19}$ ). Furthermore, an oscillating (at $\omega_{W} / N$ $=\pi \mathrm{FrSt} h / L)$ and translating source generates a wave field (OW) with a strong transversal pattern (Dupont and Voisin ${ }^{17}$ ) composed of different wave components drawn in Fig. 7(b) and calculated in Fig. 8(d). These components will have upstream and downstream horizontal phase velocities which are very different from the observation.

The only remaining mechanism able to produce horizontal isophase topology consistent with the experimental visualization is the lee wave produced by the advection of the wake coherent structures (CSLW) at a velocity $U_{\mathrm{CS}}$ smaller than the body velocity $U$. In order to demonstrate this hypothesis we measure their frequency, their wavelength and also their vertical propagation velocity.

\section{B. Wave frequency}

We measure the absolute frequency $\omega_{a}$ of the internal waves using probes fixed in the laboratory frame and the relative frequency $\omega_{r}$ using probes moving with the body. In Fig. 9, we have plotted examples of power spectra versus $\omega_{a} / N$ obtained for $h / L=0.8$ and for two Froude numbers: $\mathrm{Fr}=0.5$, where only body lee waves exist, and $\mathrm{Fr}=3$, where the short waves supersede the lee waves. The main frequency is the same in both cases and is close to $0.85 N$. So the two wave fields cannot be separated in frequency and the value of 


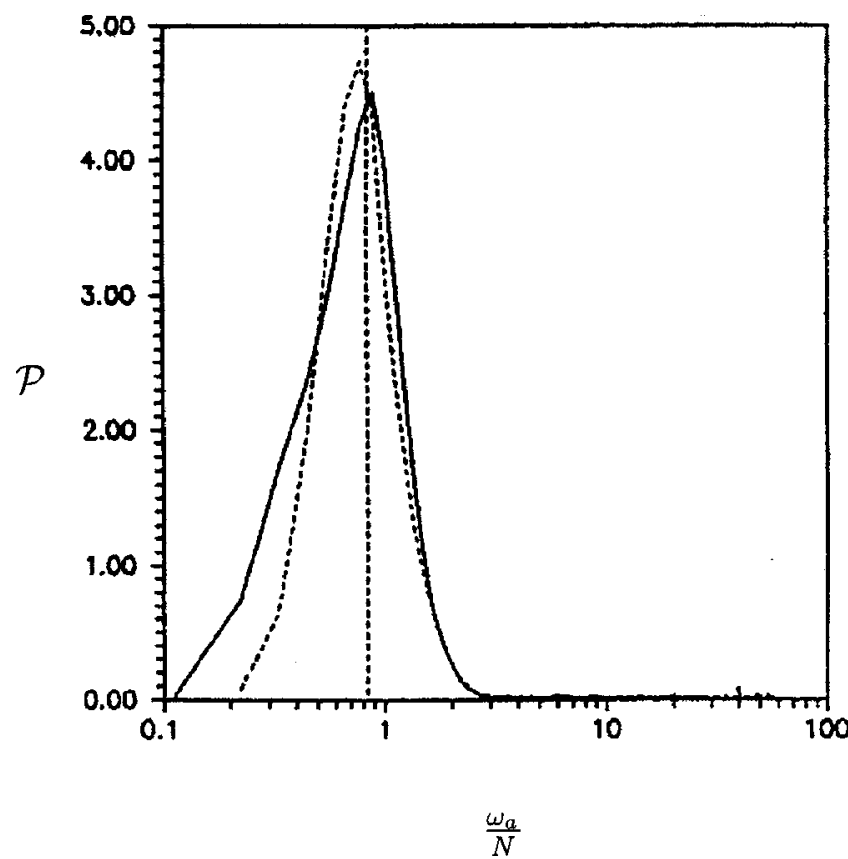

FIG. 9. Power spectra $\mathcal{P}$ of signals measuring the frequency $\omega_{a} / N \approx 0.85$ in the water frame for two experiments at Froude number $\mathrm{Fr}=1.5$ (continuous line) and $\mathrm{Fr}=3$ (dashed line).

$\omega_{a}$ is independent of the Froude number. The measured frequency would have been completely different in the case of OW generated by an oscillating body whose absolute frequency $\omega_{a}$ (in fixed frame) depends on the probe location and whose relative frequency $\omega_{r}$ (in the moving frame) is equal to $\omega_{W}$. The frequencies obtained for different $\mathrm{Fr}$ from conductivity probes moving with the topography and located away from the wake, show that $\omega_{r}$ varies with Fr and differs from $\omega_{W}$. These frequency measurements are converted into wavelengths by $\lambda_{x}=2 \pi\left(U-U_{\mathrm{CS}}\right) / \omega_{r}$ and presented on Fig. 12 together with the direct measurements of axial wavelengths from visualization similar to Figs. 5 and 6 in the next subsection.

\section{Axial wavelength}

The axial wavelength is measured by the spacing between white and black areas in the center of images similar to those on Fig. 6. Figure 10 summarizes all the measurements of axial wavelength versus time at different heights and for different Froude numbers. The wavelength is approximately independent of time and of height, but is very sensitive to the Froude number. The slow decrease of $\lambda_{x}$ with time may then be explained by the slow down of $U_{\mathrm{CS}}$ before the wake collapse.

Figure 11 shows the measured mean wavelength obtained from data plotted in Fig. 10 as filled squares for $h / L$ $=0.28$ and as filled triangles for $h / L=0.8$. It compares extremely well with the CSLW wavelength of Eq. (5) (open symbols) using the measured values of $U_{\mathrm{CS}}$ (Fig. 4). Besides, the body lee waves are also drawn as a curve corresponding to values one order of magnitude higher than the experimental data. The differences are still convincing when one compares the experimental data with the waves emitted by an

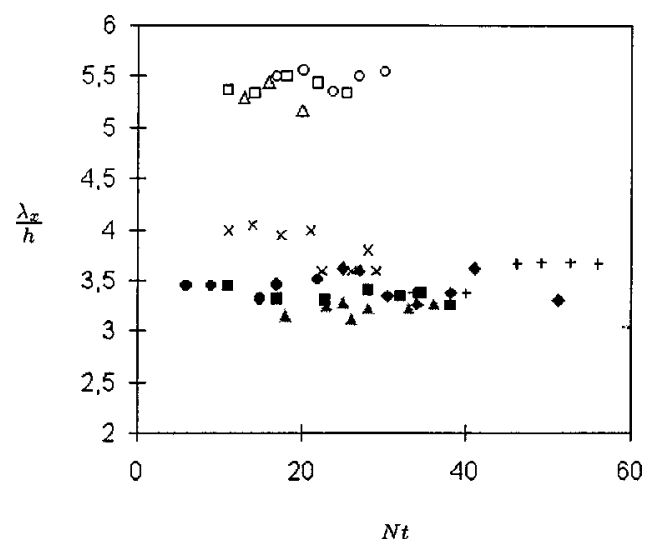

FIG. 10. Axial wavelengths $\lambda_{x} / h$ measured for the short waves visualization on the center line $y=0$ vs time $N t$ for $\mathrm{Fr}=5$ (open symbols) at $z / h$ $=5,7$ and 10, for $\mathrm{Fr}=4$ (crosses) at $z / h=5$ and 10 and for $\mathrm{Fr}=3$ (filled symbols) and $z / h=3.5,4.25,5$, and 7 .

oscillating source (OW) or by the collective effect of several coherent structure advection (RCSLW), plotted for both model as crosses on horizontal lines independent from Fr for each hill. In conclusion, on Fig. 11 it is only the CSLW theory which can account for the observed $\lambda_{x}$ values. This conclusion may be emphasized by plotting the same data as a function of coherent structure Froude number $U_{\mathrm{CS}} / N h$ (Fig. 12). The data are aligned on the theoretical law [Eq. (5)] drawn as the solid line in Fig. 12. One may notice that the coherent structure Froude number are close to unity in the measurement presented on Fig. 12 and this feature might explain the large amplitude observed for the short wave (i.e., the CSLW) in this range of parameters.

\section{Phase and group velocities}

We have seen that the apparent phase velocity of the short waves is of the order of $U_{\mathrm{CS}}$ and the measured $\lambda_{x}$ are consistent with the CSLW hypothesis (Fig. 12). This means

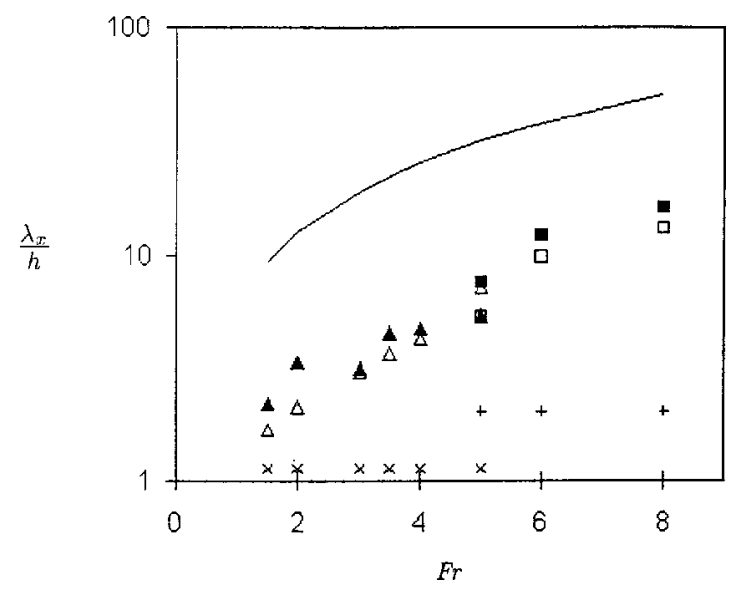

FIG. 11. Mean axial wavelength vs the Froude number. The filled points indicate the experimental results and open one of the CSLW predictions with the $h / L=0.8$ hill (squares) for $\mathrm{Fr}<5$ and with $h / L=0.28$ (triangles) for $\mathrm{Fr}>5$; the crosses are the RCSLW-OW predictions and the continuous line the value of the BLW wave component. 


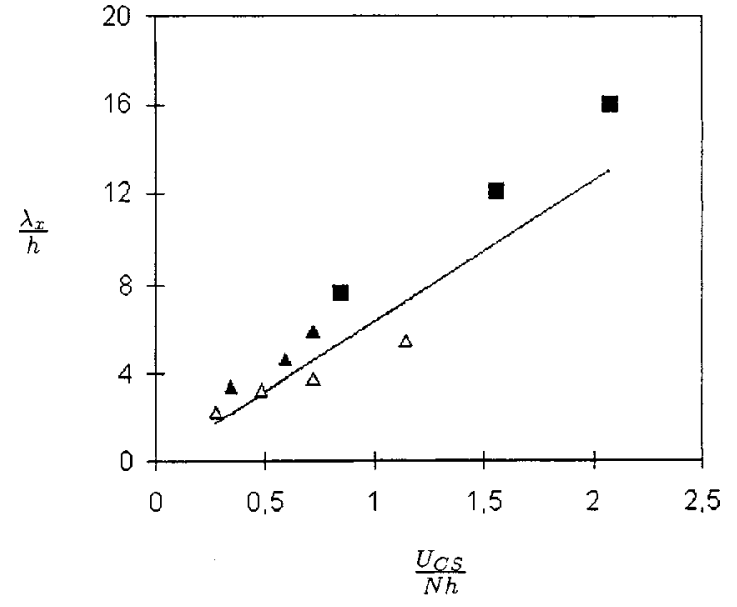

FIG. 12. Mean axial wavelength versus the advection velocity $U_{\mathrm{CS}} / N h$. The triangles indicate measurements with the $h / L=0.8$ hill and the squares with $h / L=0.28 ; \lambda$ are measured from images for open symbols and deduced from frequency values for filled symbols. The solid lines come from the linear theory of CSLW (see Sec. III D).

that the wave field is fixed with the moving coherent structure. This is a very strong criterion for the CSLW generation mechanism.

Furthermore we estimate from the previous wave visualization the emergence time $N t_{e}$ of short waves at different altitudes $z$. This time is plotted on Fig. 13 versus altitude $z / h$ and for each Froude number. For each Froude number the slope of the linear regression gives a measurements of the vertical propagation velocity (i.e., the vertical group velocity) of the short waves which propagate faster as the Froude number increases. This time correspond to the arrival of the wave front drawn in Fig. 7(d) at the observed position. Finally we reported in Fig. 14 the dimensionless vertical wave propagation velocity $(d z / d t) /(N h)$ versus the coherent structure Froude number $U_{\mathrm{CS}} /(N h)$. This relation is very close to the linear dependence, plotted as the dashed line, of the maximum vertical group velocity on the Froude number for the lee wave field of an object moving at $U_{\mathrm{CS}}: c_{g z \max }$

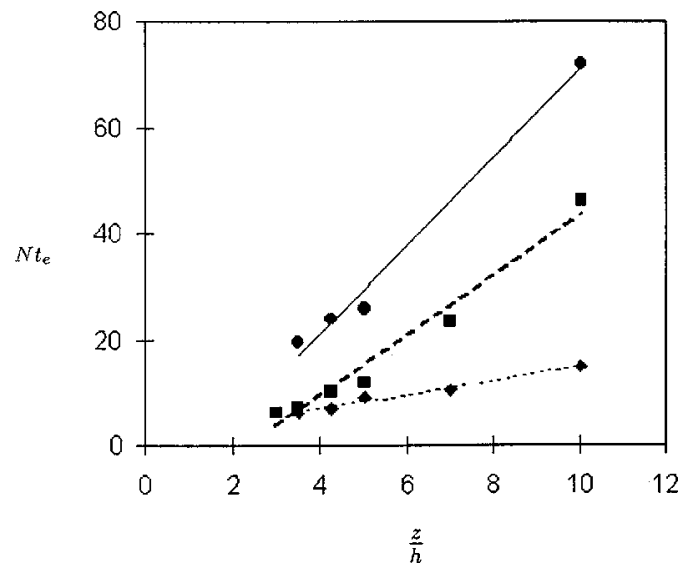

FIG. 13. Time $N t_{e}$ of emergence of internal waves generated by the wake are estimated for different Froude numbers and at different height; circles referred to $\mathrm{Fr}=1.5$, squares to $\mathrm{Fr}=3$, and diamonds to $\mathrm{Fr}=5$. The lines draw linear regression plots for each Fr and the slopes give the vertical propagation velocity $c_{g z}$.

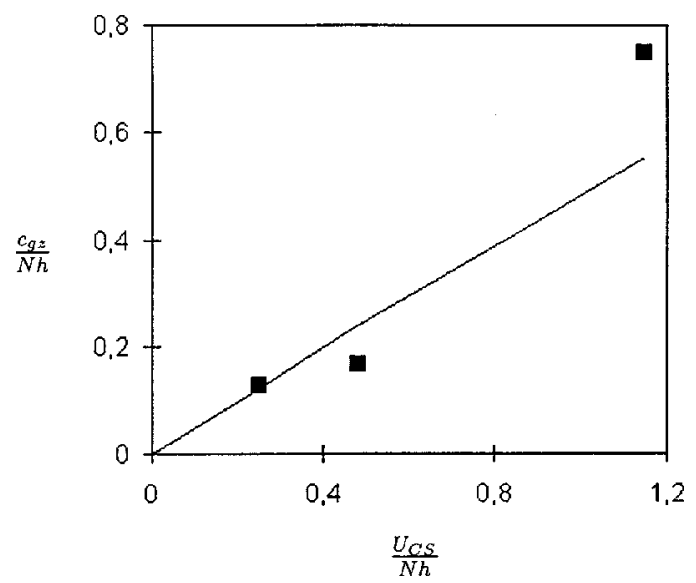

FIG. 14. Estimation of the vertical group velocity $c_{g z} / N h$ of internal waves generated by the wake versus the advection velocity $U_{\mathrm{CS}} / N h$. The lines represent the theoretical linear relation of CSLW deduced from the geometry of the propagation of these waves (see Fig. 7).

$=U_{\mathrm{CS}} / 2$ derived from the geometry of wave propagation drawn in Fig. 7(d) for a transient moving source.

\section{CONCLUSION}

A conclusion can now be drawn regarding the mechanism of generation of short waves in the wakes of Gaussian hills. Because of the hyperbolic shape of their isophases and the constancy of their wavelength in time, the short waves do not belong to the random wave field generated by the individual collapse of each vortex structure. They are clearly different from the body lee waves (including the mean wake collapse waves) whose wavelength and phase velocity are both five times larger than those observed here. The wavelength $\lambda_{x} \approx \lambda_{W}$ of $\mathrm{OW}$ is mainly independent from the Froude number variation, whereas the experimental $\lambda_{x}$ increases with Fr. Therefore, these short waves are not explained by any of the classical wave emission mechanisms previously identified in stratified wakes. We propose an alternative interpretation in term of lee waves produced by the displacement of the coherent structures themselves and develop the notion of "attached internal waves" introduced by Sysoeva and Chashechkin ${ }^{8,18}$ without any justification. Indeed, the evolution of the isophases is well predicted by the linear theory of lee waves. The phase velocity is equal to the mean advection velocity of the coherent structures. The mean axial wavelength is equal to $2 \pi U_{\mathrm{CS}} / N$. The maximum vertical group velocity of the short waves is about $U_{\mathrm{CS}} / 2$. All these aspects are typical of coherent structure lee waves (CSLW). We therefore conclude that the short waves are generated by the horizontal motion of the vortex structures as if they were solid bodies. The problem of generation of gravity wave by vortices is not at all so simple but based on observations it seems that the coherent hair pin vortices moves under their own induction and radiate gravity wave as if they were a solid body. Although not applicable, it is tempting to draw this analogy with the motion of a dipole that propagates under its own induction and generate a potential flow which is a source of internal waves. In the present case since the turn over frequency of order $U / h$ inside the coherent struc- 
ture vortex core is larger than the Brünt Väisälä frequency $N$ direct coupling does not occur and at leading order the flow inside the coherent structure does not feel the stratification. The fluid particles outside the wake are displaced by the flow around the coherent structure at a frequency $U_{\mathrm{CS}} / h$ near $N$ and therefore gravity waves are generated as they would be for a solid body motion. In our experimental demonstration, there is a lack of model able to predict the amplitude of such waves and it gives rise to an important question: Between the steady lee wave field and the impulsive wave field generated by a transient source (the coherent structure, for example) moving during a characteristic time $\tau$ at a mean velocity $U_{\mathrm{CS}}$, which is the dominant one? Considering this problem, Stevenson ${ }^{20}$ and Voisin ${ }^{21}$ have studied the resulting surfaces of constant phase and separated the space into two domains corresponding to each wave field: The lee wave field is inside a sphere of diameter $U_{\mathrm{CS}} . \tau$ and the impulsive one outside. Sturova ${ }^{22}$ has studied experimentally the wave amplitude in the case of a transient moving sphere for $\mathrm{Fr}=33$ and found a maximum at the spherical boundary between the two wave field. But our results demonstrate the need of a complete understanding of these kind of transient sources.

\section{ACKNOWLEDGMENTS}

This work was achieved at the research center of the French Met Office. We wish to thank the whole SPEA team and in particular Mr. Perrier for their enthusiasm and efficiency. We also thank E. J. Hopfinger, P. Bonneton, and B. Voisin for their scientific support. Our collaboration was under the hospice of the GdR M.F.G. of the CNRS (French National Research Organization) and one of us (J.-M.C.) wants to thank Buck Dany for inspiration.

${ }^{1}$ J.-T. Lin and Y.-H. Pao, "Wakes in stratified fluids," Annu. Rev. Fluid Mech. 11, 317 (1979).

${ }^{2}$ G. E. Merrit, "Wake growth and collapse in stratified flow," AIAA J. 12, 940 (1974).

${ }^{3}$ A. H. Shooley and B. A. Hughes, "An experimental and theoretical study of internal waves generated by the collapse of a two-dimensional mixed region in a density gradient,” J. Fluid Mech. 51, 159 (1972).

${ }^{4}$ P. Bonneton, J.-M. Chomaz, and E. J. Hopfinger, "Internal waves produced by the turbulent wake of a sphere moving horizontally in a stratified fluid," J. Fluid Mech. 254, 23 (1993).

${ }^{5}$ H. E. Gilreath and A. Brandt, "Experiments on the generation of internal waves in a stratified fluid," AIAA J. 23, 673 (1985).

${ }^{6}$ B. Voisin, "Internal wave generation by turbulent wakes," in Proc. Meeting-Workshop on Mixing in Geophysical Flows, edited by J. M. Redondo and O. Métais (CIMNE, Barcelona, 1996).

${ }^{7}$ Q. Lin, D. L. Boyer, and H. J. S. Fernando, "Internal waves generated by the turbulent wake of a sphere," Exp. Fluids 15, 147 (1993).

${ }^{8}$ E. Ya. Sysoeva and Yu. D. Chaschechkin, "Vortex system in the stratified wake of a sphere," Fluid Dyn. 15, 378 (1991).

${ }^{9}$ G. Oster, "Density gradients," Sci. Am. 213, 70 (1965).

${ }^{10}$ J.-M. Chomaz, P. Bonneton, A. Butet, E. J. Hopfinger, and M. Perrier, "Gravity wave patterns in the wake of a sphere in a stratified fluid," in Turbulence and Coherent Structures, edited by O. Métais, and M. Lesieur (Kluwer Academic, Dordrecht, 1991), pp. 489-503.

${ }^{11}$ Y. Kadri, P. Bonneton, J. M. Chomaz, and M. Perrier, "Stratified flow over three-dimensional topography," Dyn. Atmos. Oceans 23, 321 (1996).

${ }^{12}$ Q. Lin, D. L. Boyer, and H. J. S. Fernando, "The vortex shedding of a streamwise-oscillating sphere translating through a linearly stratified fluid," Phys. Fluids 6, 239 (1994).

${ }^{13}$ C. W. van Atta and E. J. Hopfinger, "Vortex ring instability and collapse in a stably stratified fluid," Exp. Fluids 7, 197 (1989).

${ }^{14}$ M. J. Lighthill, Waves in Fluids (Cambridge University Press, Cambridge, England, 1978).

${ }^{15}$ J. W. Miles, "Internal waves generated by a horizontally moving source," Geophys. Fluid Dyn. 2, 63 (1971).

${ }^{16}$ E. J. Hopfinger, J.-B. Flor, J.-M. Chomaz, and P. Bonneton, "Internal waves generated by a moving sphere and its wake in a stratified fluid," Exp. Fluids 11, 255 (1991).

${ }^{17}$ P. Dupont and B. Voisin, "Internal waves generated by a translating and oscillating sphere," Dyn. Atmos. Oceans 23, 289 (1996).

${ }^{18}$ E. Ya. Sysoeva and Yu. D. Chaschechkin, "Vortex structure of a wake behind a sphere in a stratified fluid," J. Appl. Mech. Tech. Phys. 27, 190 (1986).

${ }^{19}$ P. Dupont, "Ondes internes engendrées par une source oscillante en mouvement," Ph.D. thesis (in French), Grenoble (1995).

${ }^{20}$ T. N. Stevenson, "The phase configuration of internal waves around a body moving in a density stratified fluid," J. Fluid Mech. 60, 759 (1973).

${ }^{21} \mathrm{~B}$. Voisin, "Internal wave generation in uniformly stratified fluids. Part 2. Moving point sources," J. Fluid Mech. 261, 333 (1994).

${ }^{22}$ I. V. Sturova, "Internal waves generated in an exponentially stratified fluid by an arbitrarily moving source," Fluid Dyn. 15, 378 (1980). 